\title{
A Human Novel Gene DERPC Located on 16q22.1 Inhibits Prostate Tumor Cell Growth and Its Expression Is Decreased in Prostate and Renal Tumors
}

\author{
Mei Sun, ${ }^{1}$ Lanfeng Ma, ${ }^{1}$ Linda Xu, ${ }^{1} \mathrm{Jia} \mathrm{Li}^{1}{ }^{1}$ Wei Zhang, ${ }^{2}$ Gyorgy Petrovics, ${ }^{1}$ Mazen Makarem, ${ }^{1}$ \\ Isabell Sesterhenn, ${ }^{2}$ Mei Zhang, ${ }^{3}$ E. Joan Blanchette-Mackie, ${ }^{3}$ Judd Moul, ${ }^{1,4}$ Shiv Srivastava, ${ }^{1}$ \\ and Zhiqiang Zou ${ }^{1}$ \\ ${ }^{1}$ Center for Prostate Disease Research, Department of Surgery, Uniformed Services University of the Health \\ Sciences, Rockville, MD, USA \\ ${ }^{2}$ Armed Forces Institute of Pathology, Department of Genitourinary Pathology, Washington, DC, USA \\ ${ }^{3}$ Section Lipid Cell Biology, Laboratory of Cell Biochemistry and Biology, National Institute of Diabetes \\ and Digestive and Kidney Diseases, National Institutes of Health Bethesda, MD, USA \\ ${ }^{4}$ Urology Service, Department of Surgery, Walter Reed Army Medical Center, Washington, DC, USA \\ Accepted August 27, 2002
}

\begin{abstract}
Background: Deletion of chromosome $16 \mathrm{q}$ is frequently associated with diverse tumors. Numerous studies strongly suggest the presence of one or more tumor suppressor genes on chromosome 16q22 to l6qter including the widely studied cadherin gene family. However, the specific tumor suppressor genes residing in this region need better definition and characterization.

Material and Methods: Standard molecular biology approaches have been used to clone and characterize the $D E R P C$ cDNA and its protein product on chromosome 16q22.1. Northern blotting was used to define the expression pattern in a multiple human tissue blots. $D E R P C$ expression was examined in multi-tumor array (Clontech, CA, USA) dot blot as well as in laser capture microdissection (LCM) derived prostate cancer (CaP) specimens by quantitative RT-PCR. Western blot analysis and a fluorescent microscopy were used to characterize the molecular size and the cellular location of green
\end{abstract}

fluorescent protein (GFP)-tagged DERPC fusion proteins. A colony formation assay was conducted to determine the effects of DERPC expression on tumor cell growth.

Results: A novel gene DERPC (Decreased Expression in Renal and Prostate Cancer) was identified and characterized. DERPC encoded a strong basic, proline- and glycinerich nuclear protein. DERPC was ubiquitously expressed, with abundant expression in kidney, skeletal muscle, testis, liver, ovary, and heart and moderate expression in prostate. DERPC expression was reduced in renal $(67 \%)$ and prostate tumors (33\%). Expression of DERPC has inhibitory potential on CaP cell growth. Further, overexpression of DERPC in LNCaP cells caused alterations of nuclear morphology.

Conclusion: This study suggests that decreased expression of DERPC may be implicated in tumorigenesis of renal and CaPs.

\section{Introduction}

Prostate cancer (CaP) is the second leading cause of cancer-related deaths among men in the United States (1). The molecular determinants in the development and progression of this disease are poorly understood. In recent years, increasing attention has been directed to finding genes that play a causal role in the process of tumorigenesis, such as tumor suppressor genes (TSGs) and oncogenes. Chromosomal loci, which are associated with sporadic as

Address correspondence and reprints requests to: Zhiqiang Zou, Center for Prostate Disease Research, 1530 East Jefferson Street, Rockville, MD 20852, USA. Phone: (240) 453-8932;

fax: (240) 453-8912; e-mail: zzou@cpdr.org.

The opinions and assertions contained herein are the private views of the authors and are not to be considered as reflecting the views of the U.S. Army or the Department of Defense. well as inherited CaPs, are being intensely analyzed for putative TSGs or proto-oncogenes. High frequency of allelic losses on 8p2l-22, 10q23-25, $7 \mathrm{q} 31$, and 16q24 loci have been shown in CaP. There has been extensive work from several laboratories on the characterization of chromosome 8p21-22 locus, but the identity of tumor suppressor gene has remained elusive (2-7). NKX3.1 on the 8 p21 locus has been suggested as a candidate TSG. However, no tumor-associated mutations have been reported (6). PTEN/MMACI on chromosome 10q25 has been discovered as a TSG frequently altered in advanced CaP $(8,9)$. ST7 on chromosome $7 \mathrm{q}$ has been identified to be mutated in prostate and other cancers (10). Mutations of the KLF6 gene on chromosome $10 \mathrm{p}$ have also been recently reported in sporadic CaPs (11). On the other hand, gains of chromosome 8q24 harboring c-myc and prostate 
stem cell antigen (PSCA) genes have also been shown in CaP (12). Studies utilizing comparative genomic hybridization (CGH) have shown frequent losses of novel chromosomal loci including $2 \mathrm{q}, 5 \mathrm{q}$, and $6 \mathrm{q}$ and gains of $11 \mathrm{p}, 12 \mathrm{q}, 3 \mathrm{q}, 4 \mathrm{q}$, and $2 \mathrm{p}$ in $\mathrm{CaP}$ (13). Our own study shows the high rate of LOH on 6q16 using laser capture microdissection (LCM)derived CaP specimens (14). Chromosome regions $1 \mathrm{q}, 13 \mathrm{q}, 17 \mathrm{p}, 20 \mathrm{q}$, and $\mathrm{Xq}$ have been linked to familial CaP $(15,16)$. Recent reports have described germline mutations of the ELAC2 and RNASEL1 in a small subset of CaP-prone families (17-19). Thus, identification and analysis of candidate genes in CaP-associated chromosomal deletion region have promise in defining CaP-specific TSGs and/or oncogenes.

Deletions and allelic imbalance (AI) on human chromosomal region 16q21-24 have been detected by various genetic approaches in human $\mathrm{CaP}$, as well as other cancers, including breast cancer, Wilm's tumors, hepatocellular carcinoma, and acute myeloid leukemia (20-26). These observations suggest that this region may harbor multiple tumor suppressor loci, which might be implicated in the development of $\mathrm{CaP}$ and other malignancies (27). In the search for tumor suppressor genes that are the targets of loss of heterozygosity on 16q, the E-cadherin gene, $C D H 1$, was unveiled by the identification of mutations and/or diminished expression in CaP and other malignancies (28-30). Investigations are still in progress to find the target genes from this region in prostate tumors. We report here the cloning and the characterization of a novel gene named DERPC that locates on 16q22.1, $260 \mathrm{kbp}$ apart from the $C D H 1$ locus. The expression of DERPC was found to be significantly decreased in renal tumors, as well as in prostate tumors. DRPEC encodes a nuclear protein with cell growth inhibitory characteristics. We suggest that decreased expression of DRPEC may play a role in the process of tumorigenesis in renal and prostate tumors.

\section{Materials and Methods \\ Cell Culture}

Prostate tumor cell lines DU145, LNCaP, and PC3 and kidney embryonic cells 293 cells were obtained from American Type Culture Collection (ATCC, Rockville, MD, USA) and were maintained in growth medium recommended by the supplier.

\section{Full-Length DERPC cDNA Sequence}

IMAGE clones of EST were purchased from ATCC and sequenced using primers from $5^{\prime}$ and $3^{\prime}$ ends of the vector followed by internal primers designed from the initial sequence data. Full-length cDNA was obtained by RT-PCR. DNA sequencing was performed using the Amplicycle sequencing kit from
Applied Biosystems (Foster City, CA, USA) on 3100 DNA Genetic Analyzer.

\section{Northern and Dot Blot Analysis}

Northern blots containing multiple human tissue mRNA and multi-tumor array were purchased from Clontech. The probe was labeled with ${ }^{32} \mathrm{P}$-dCTP by random priming (Stratagene) following the manufacturer's directions. Hybridization was carried out at $68^{\circ} \mathrm{C}$ for $12-14 \mathrm{hr}$ in ExpressHyb Solution (Clontech) containing $1 \times 10^{6} / \mathrm{ml}{ }^{32} \mathrm{P}-$ labled probe followed by stringent washing in $0.1 \%$ SDS, 2 XSSC at room temperature, and $0.1 \%$ SDS, 0.1 XSSC at $68^{\circ} \mathrm{C}$. The membranes were exposed to a Kodak XR film for autoradiography.

\section{Quantitative RT-PCR}

Matched normal and tumor samples were obtained by LCM of frozen sections derived from radical prostatectomy specimens. Total RNA was extracted from cells by RNAzol method (Gibco BRL). Total RNA was reverse transcribed and subjected to quantitative PCR. The quantitative PCR was performed using SYBR green PCR kit (Applied Biosystems) on PE-7700 Sequence Detection System. Ct value obtained from gene specific primers was used to calculate the expression difference between normal and tumor samples after calibration with the $\mathrm{Ct}$ value from housekeeping gene GAPDH.

\section{Bioinformatics}

The cDNA sequence was searched for homology and similarity against GenBank using BLAST programs, including BLASTN, BLASTX, and BLASTP (available from URL: http://www.ncbi.nlm.nih.gov). Predicted protein sequence was analyzed by webbased sofware: TMPred (available from URL: http:// www.ch.embnet.org/software/TMPRED_form.html), ProfileScan (available from URL: http://www.isrec. isb-sib.ch/software/PFSCAN_form.html), ScanProsite (available from URL: http://expasy.cbr.nrc.ca/tools/ scnpsitl.html), and Interpro (available from URL: http://www.ebi.ac.uk/interpro/). We performed alignment of protein sequences using ClustalW Multiple Sequence Alignment (@BCM) (available from URL: http://dot.imgen.bcm.tmc.edu:9331/multi-align/Options/clustalw.html).

\section{Construction of Expression Plasmid}

Wild-type DERPC cDNA was obtained by RT-PCR amplification of the entire open reading frame (ORF) of DERPC from human prostate Marathon-Ready cDNA (Clontech) using forward primer (TGGTGACAGCACTCATCAAAGACAAG) and reverse primer (GGCTGGAGATCCTTTCTCTCAAGG), followed by cloning into PCR-blunt II TOPO vector (Invitrogen). DERPC was excised from PCR-blunt II by $E c o R$ I digestion and inserted into mammalian 
expression vector pcDNA3.1(+) (Invitrogen) to generate pcDNA-DERPC sense and antisense constructs.

The construct encoding green fluorescent protein (GFP)-DERPC was generated by in-frame insertion of $D E R P C$ from PCR-blunt II TOPO into pEGFP-Cl at EcoR I site. A spacer peptide encoded by the multiple cloning sites of the GFP vector YKSGLRSRAQASNSPFGDSTHQRQDPFQNPPQAHYHQRPQES was included between GFP and DERPC. To generate the construct encoding DERPC-GFP, we introduced a 7-glycine linker in-frame followed by a PinA I site into 3' end of DERPC to replace its stop codon by PCR. PCR product of DERPC was then cut by KpnI and PinAI and inserted in-frame into the same sites in pEGFPN1. An amino acid sequence GGGGGGGQPVAT containing the glycine linker and the codons derived from the multiple cloning sites of the GFP vector was created between DERPC and GFP. All constructs were verified by DNA sequencing.

\section{Transfection}

Cells were seeded at approximately $40-60 \%$ confluence in 2-well Lab-Tek chambered slides 18-24 hr before transfection. Transient transfection was carried out using FuGene6 reagent (Boehringer Mannheim) following the guidelines supplied by the manufacturer. Briefly, cells were incubated with DNA/FuGene6 mixture in OPTI Medium (Invitrogen) for 2-5 hr followed by culturing in medium containing an appropriate concentration of FBS. Cells were fixed 48-72 hr after transfection with 3\% paraformaldehyde for $30 \mathrm{~min}$ and stained with DAPI.

\section{Confocal Microscopy}

Confocal images were obtained with an LSM410 confocal microscope (Carl Zeiss Inc., Thornwood, USA, NY) equipped with an Omnichrome kryptonargon laser (Melles Griot, Carlsbad, CA, USA) with excitation wavelengths of $488 \mathrm{~nm}$ for EGFP and an argon ion laser (Coherent, Santa Clara, CA) with excitation wavelength of $351 \mathrm{~nm}$ for DAPI staining.

\section{Immunoblotting}

Cells were lysed in $1 \times$ SDS lysis buffer and boiled for $10 \mathrm{~min}$. The extract was centrifuged at $10,000 \mathrm{~g}$ for $10 \mathrm{~min}$ and the supernatant was aliquoted and stored at $-70{ }^{\circ} \mathrm{C}$. Total protein $(100 \mu \mathrm{g})$ from each sample was separated on $10 \%$ SDS-PAGE gel electrophoresis. The proteins were transferred to nitrocellulose membrane (Novex) and Western blot analysis was performed using an ECL blot kit (Amersham) according to the manufacturer's instruction. Mouse monoclonal antibody (mAb) against GFP (Boehringer Mannheim) was used at a 1:1000 dilution to detect the tagged DERPC.

\section{Colony Formation Assay}

Cells $\left(2 \times 10^{5}\right)$ were seeded in a $60-\mathrm{mm}$ dish 1 day before transfection. The expression plasmid DNA
$(5 \mu \mathrm{g})$ was transfected into tumor cells using TransFast reagent (Promega, Madison, WI, USA) according to the protocols provided by the manufacturer. The cells were then cultured in medium containing $400-500 \mu \mathrm{g} / \mathrm{ml}$ of geneticin (G-418) for $2-4$ weeks. Cell colonies were stained with crystal violet.

\section{Results}

\section{Isolation and Characterization of DERPC}

An EST sequence of DERPC, localizing to chromosome 16q22.1, was initially selected from Serials Analysis of Gene Expression (SAGE) library of genes expressed in LNCaP cells (31). The notable chromosomal localization of DERPC on 16q22.1, which is frequently altered in various types of human tumors, caused us to further investigate DERPC. A 2.9-kbp cDNA sequence of DERPC was assembled by sequence analysis of EST IMAGE clones and PCR-derived DNA fragment from normal prostate cDNA (Fig. 1B). Complete cDNA sequence revealed a single open reading frame (ORF) of $1575 \mathrm{nu}-$ cleotides encoding a protein of 524 amino acids with an approximate molecular mass of $51 \mathrm{kDa}$. Then $D E R P C$ CDNA sequence was compared to GenBank using BLASTN, which identified the human chromosome 16 clone RP11-123C5 (GenBank accession number AC009027.10). We constructed a physical map of this region based on the Ensembl Human ContigView (available from URL: http://www. ensembl.org/Homo_sapiens) (Fig. 1A). The genomic structure of DERPC was determined by aligning the cDNA sequence against the genomic sequence of chromosome 16 clone RP11-123C5. DERPC is composed of four exons that span $14.5 \mathrm{kbp}$ of genomic DNA. However, the entire ORF was localized in exon 4 (Fig. 1A).

The DERPC is a highly basic protein with PI of 12.5. It contains abundant proline $(20 \%)$ and glycine $(18 \%)$ residues. Structural analysis of the amino acid sequence using the TMpred membranespanning-prediction program (TMpred-Prediction of Transmembrane Regions and Orientation) revealed absence of significant transmembrane domain in the DERPC encoded protein, suggesting that $D E R P C$ encodes a nonmembrane protein. Comparison of the amino acid sequence with known protein motifs and patterns at ProfileScan identified a prolineglycine rich domain (AA4-467). Furthermore, the predicted DERPC peptide contained 11 repeats of the sequence PXXP (where P represents proline and $X$ any amino acid), which is known to bind to the SH3 domain (Fig. 1B). We therefore predict that $D E R P C$ encodes a proline-glycine rich protein potentially interacting with $\mathrm{SH} 3$ containing proteins implicated in signal transduction.

The predicted DERPC peptide shared an $86 \%$ sequence homology with a human hypothetical protein (GenBank accession: xm_093070) and an 82\% mouse homolog (accession: BC023107) (Fig. 2). The 
human hypothetical protein contained additional 47 amino acids at the $\mathrm{N}$-terminus derived from a predicted exon. The human homologous gene is localized to chromosome $\mathrm{X}$ and encoded 560 amino acids. Because the human hypothetical protein is predicted by automated computational analysis using the gene prediction method, GenomeScan computer program, its true identity remains to be verified. Nevertheless, it is possible that DERPC and the hypothetical protein belong to the same family of proteins. The mouse homolog of DERPC is derived from a cDNA library. It is likely that this gene represents the mouse homolog of DERPC. To define the location of mouse homolog DERPC on mouse genome, we searched mouse genome database using Genomic Blast, which identified two mouse DNA contigs (NW_000349.1 on mouse chromosome 8 and NW_000102.1 on chromosome 15). Comparison of the known gene loci surrounded the mouse DERPC to the human revealed that the nearest mouse Cdhl locus on chromosome 8 is similar to the corresponding region of human DERPC. The presence of the Cdhl locus confirms and extends to the region of synteny between human chromosome 16q22.1 and the mouse chromosome 8 .

\section{Decreased Expression of DERPC in Renal and Prostate Tumors}

Northern blot analysis of multiple human tissue blots showed a 3.0-kb DERPC transcript, which was ubiquitously expressed, with abundant expression in kidney, skeletal muscle, testis, liver, ovary, and heart, and moderate expression in prostate (Fig. 3A).
To investigate the correlation of DERPC expression with tumors, we probed DERPC CDNA to multitumor array blot. We found a significantly decreased DERPC mRNA in $67 \%(10 / 15)$ of renal tumors compared to matched normal tissues (Fig. 3B).

$D E R P C$ expression was further analyzed in LCMderived CaP specimens by quantitative RT-PCR. $D E R P C$ expression was found to be decreased more than 4 -fold in $32 \%(12 / 38)$ of prostate tumors as compared to their normal counterparts (Table 1). These studies suggest that decreased expression of DERPC might play a role in renal and prostate tumorigenesis.

\section{DERPC Resides in the Nucleus and Alters Morphology of LNCaP Cells}

To analyze DERPC cellular location, we generated two constructs by fusing GFP at the $\mathrm{N}$ - and C-termini of DERPC, respectively. Expression of the fusion proteins was monitored by both Western blot analysis and confocal microscopy in transiently transfected CaP cell lines, PC3 and LNCaP. To verify whether the fusion proteins were expressed properly, fusion proteins were analyzed with the antiGFP antibody on Western blot. PC 3 cells were transfected with the GFP-tagged DERPC constructs and lysed $48 \mathrm{hr}$ after transfection. An 80- or 83-kDa band was detected in the DERPC-GFP or GFP-DERPC transfected cells with the anti-GFP antibody, which was the expected molecular mass for the chimeric proteins. No significant degraded products were detected in cells expressing chimeric proteins. Parental vectors pEGFP-Nl and pEGFP-Cl expressed 27 and $30 \mathrm{kDa}$ of GFP, respectively (Fig. 4A).

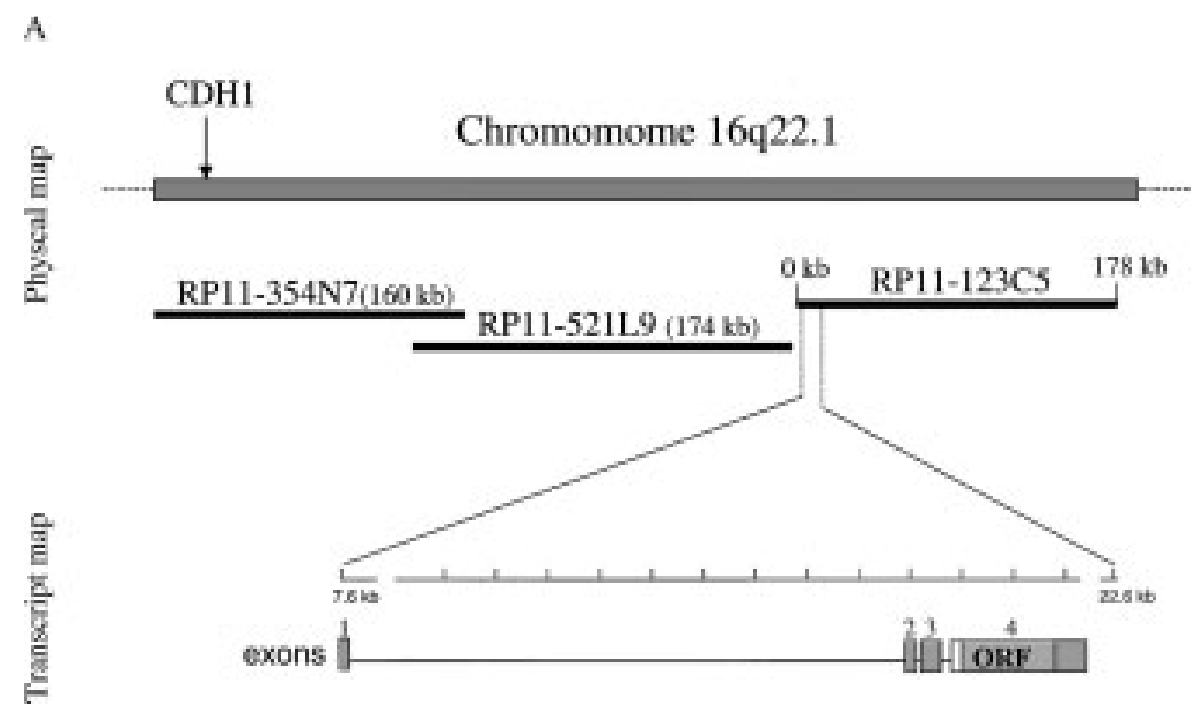

Fig. 1. Identification of DERPC gene. (A) Physical and transcript map of the DERPC gene region. Physical map shows a 500-kb segment of human chromosome 16q22.1 near the CDH1 locus. The region is covered by three contigs: RP11-354N7 (AC009082), RP11-521L9 (AC009137), and RP11-123C5 (AC009131). The transcript map of DERPC shows four exons that span 14.5 kb of genomic DNA. The ORF is present in exon 4. The scale of the transcript map is in reference to the 178-kbp scale of BAC RP11-123C5. (B) The nucleotide and deduced amino acid sequence of DERPC. The composite $2934 \mathrm{bp}$ of DERPC cDNA contains a single 1575-bp ORF that predicts a protein of 524 amino acids (AF 525164). The first ATG at nucleotide positions $485-487$ and the polyadenylation signal AATAAA are shown in bold. The 11 PXXP motifs are underlined. 
B

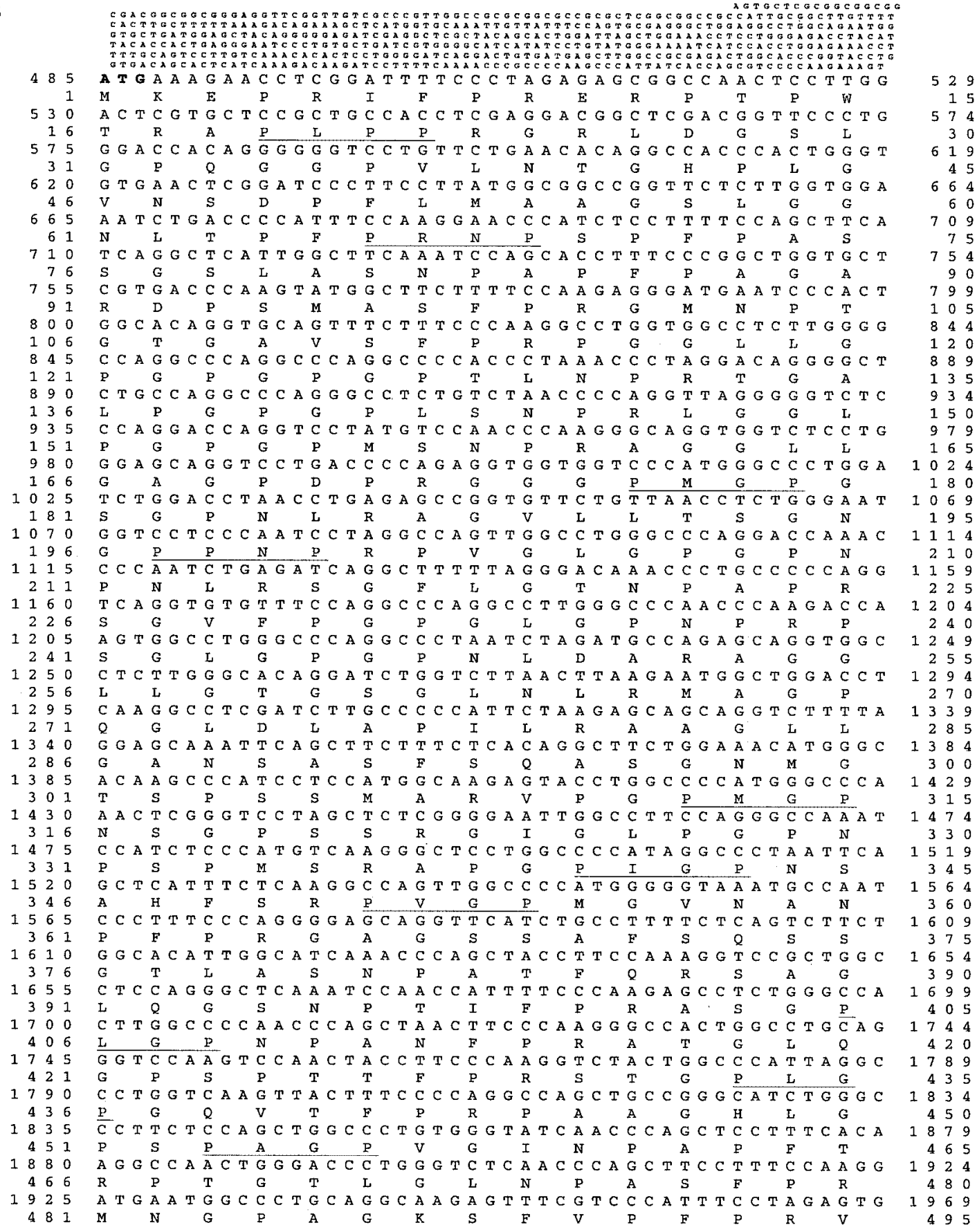

Fig. 1. (Continued)

Confocal microscopy showed that both GFPtagged DERPC chimeric proteins are diffusely spread across the whole nucleus but excluded from the nucleolus (nucleoplasmic) (Figs. 4B and 4D) counterstained with DAPI (Figs. 4C and 4E) in PC3 cells transfected with either GFP-DERPC or DERPC-GFP constructs. Interestingly, a prompt change of nuclear morphology was observed in LNCaP cells transfected with GFP-DERPC $72 \mathrm{hr}$ after transfection (Fig. 4F). The nuclei were enlarged and lobulated in transfected cells, compared with LNCaP cells transfected with empty GFP vector (Fig. 4G). However, the TUNNEL assay did not reveal apoptosis (data not shown).

Expression of DERPC Inhibits Cell Growth of PC3 Cells

To determine the possibility that DERPC expression may inhibit tumor cell growth, we conducted a colony formation assay. The pcDNA-DERPC sense, pCDNA-DERPC antisense constructs, and empty 


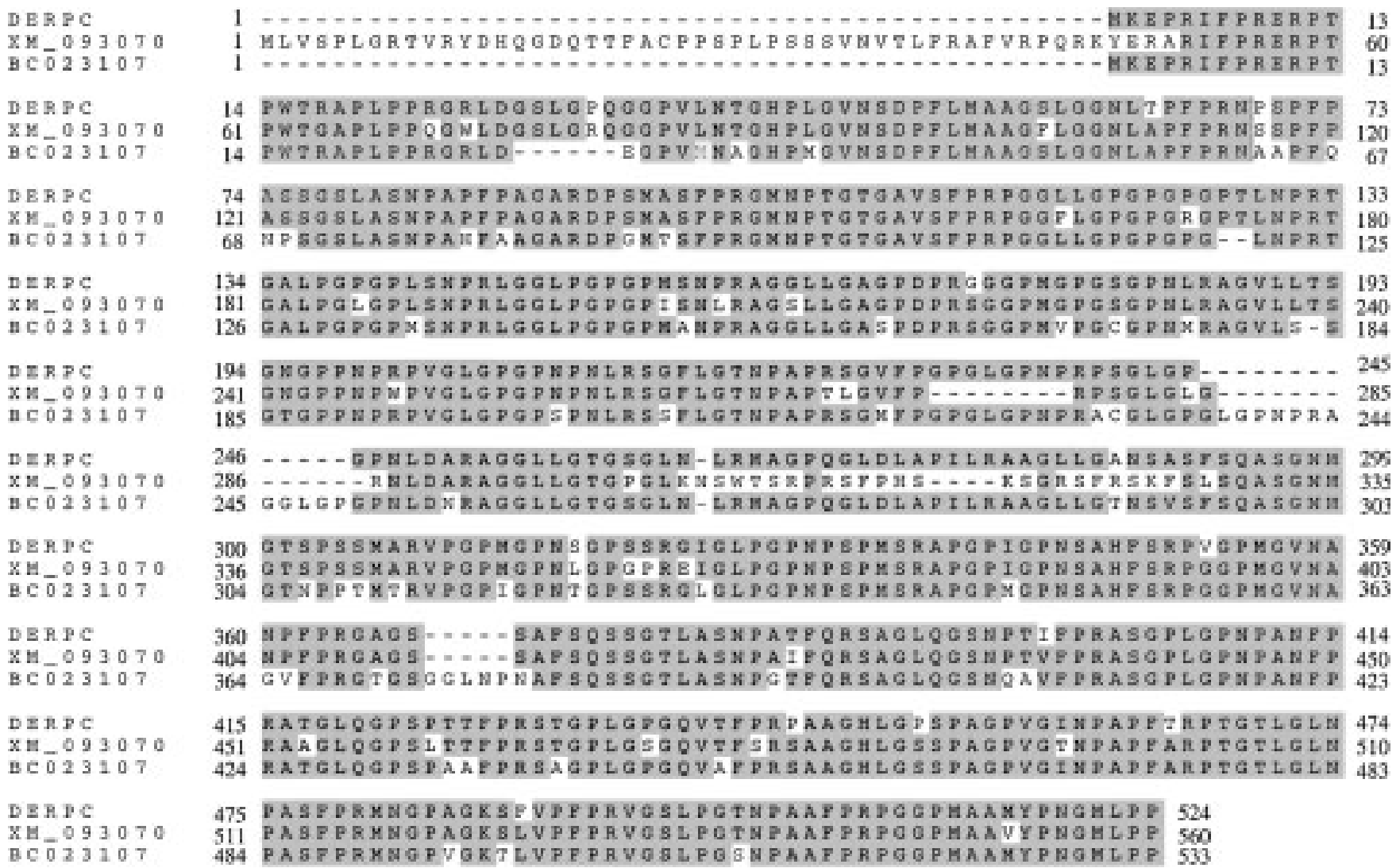

Fig. 2. Alignment of the predicted DERPC protein sequence and related amino acid sequences. Clustal W algorithm was used for the alignment. DERPC, human DERPC peptide (524-amino-acid); xm_093070, human hypothetical protein (GenBank accession no: $x \mathrm{x}_{-}$093070) predicted from genomic sequence; BC023107, mouse hypothetical protein predicted from a cDNA clone (GenBank accession: BC023107) identified by a large-scale cDNA sequencing approach. Identical residues are shaded. The gaps inserted by the Clustal W program are denoted by broken lines.

pcDNA3.1(+) vector carrying the neoresistant gene were transfected into PC3 cells, which were then maintained in medium containing G418. The drugresistant colonies were counted 2-4 weeks after transfection. A representative experiment is shown in Figure 5, which demonstrated that DERPC significantly suppressed the growth of PC 3 cells. Results from two separate experiments showed that the colony numbers were reduced by $40-50 \%$ compared to the vector and DERPC antisense controls. These data suggest that expression of DERPC inhibited prostate tumor cell growth.

\section{Discussion}

Despite the recent advance in the search for tumor suppressor genes in $\mathrm{CaP}$, the target genes in the well-defined chromosome deletion regions remain to be identified. We used an approach of selectively analyzing candidate genes in the frequent chromosomal deletion region such as $16 \mathrm{q}$ to search for potential tumor suppressor genes in CaP. A candidate tumor suppressor gene that we have named DERPC located on chromosome 16q22.1 was identified and characterized. Our study demonstrated that DERPC expression was decreased in prostate tumors, as well as in renal tumors. Overexpression of DERPC decreased the number of drug-resistant colonies of prostate tumor cell line, PC3 cells, by stable transfection in a colony inhibition assay. A prominent change of nucleus was found when GFP-DERPC fusion protein was transiently expressed in LNCaP cells. This evidence suggests that DERPC might have a potential tumor-suppressing function.

The presence of a gene mutation is still the most convincing evidence that a gene is a tumor suppressor gene. However, inactivation of a tumor suppressor gene occurs with multiple mechanism including mutation of allele and LOH of remaining allele or silencing of expressing by methylation of promoter region of a gene. Epigenetic silencing of a tumor suppressor gene is often induced by hypermethylation of the promoter region. Hypoinsufficiency occurs when only one allele of the tumor suppressor gene is inactivated. We approached to analyze gene expression as well as gene mutation for the initial screening of candidate tumor suppressor genes in frequently altered, tumor-associated chromosomal regions. Chromosome $16 \mathrm{q}$ has long been suspected of containing multiple tumor suppressor genes. DERPC in close proximity to 


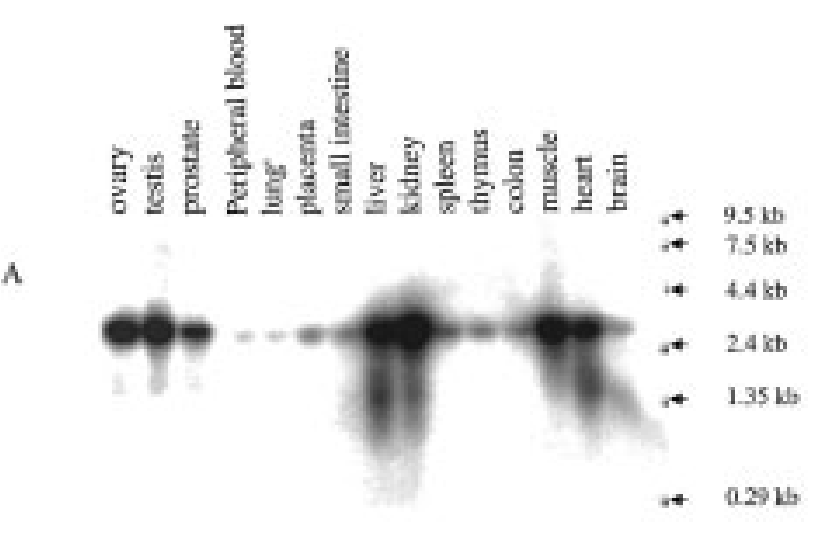

B

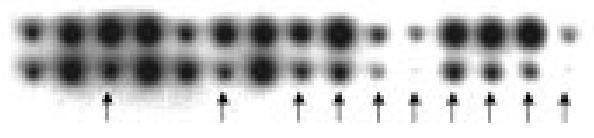

normal

renal tumor:

Fig. 3. Expression analysis of DERPC gene. (A) Northern blot analysis of the DERPC gene. Commercially available human multiple tissue blots were hybridized with a cDNA probe spanning the entire coding sequence of DERPC. A band of 3.0-kbp was detected in all tissues tested, with the abundant expression in kidney, skeletal muscle, testis, liver, ovary, and heart, and moderate expression in prostate. (B) Dot blot analysis of DERPC expression in renal tumor. Commercially available human multi-tumor array was hybridized with DERPC cDNA probe. The decreased expression of DERPC in tumors compared to matched normal tissues was denoted by arrows.

E-cadherin warranted further evaluation. First, expression of DERPC was investigated in paired normal and tumor samples. DERPC expression was decreased in more than half of the renal tumors in

Table 1. Quantitative RT-PCR analysis of DERPC expression in prostate tumors

\begin{tabular}{lc}
\hline \multicolumn{1}{c}{ DERPC Expression } & $\%(N)$ \\
\hline Tumor lower than normal & $32 \%(12 / 38)$ \\
Tumor equal normal & $58 \%(22 / 38)$ \\
Tumor higher than normal & $10 \%(4 / 38)$ \\
\hline
\end{tabular}

Comparison of DERPC expression in prostate normal and tumor cells. Prostate tumor and normal cells were obtained by LCM of frozen section of ex vivo biopsy from radical prostatectomy specimens. Total RNA was isolated from LCM-derived cells and reverse transcribed. Quantitative real-time PCR was performed using SYBR green kit on PE-7700 Sequence Detection System. The CT value was used for estimation of DERPC expression after calibration to the housekeeping gene CT value of GAPDH. The experiment was repeated two times with two sets of PCR primers: Set 1, forward, TGCAAATTGTTATTTCCAGTGCGA, Reverse, CAGGGATTCCCTCAGTGGTGT; set 2, Forward, TGCAAATTGTTATTTCCAGTGCGA, Reverse, TCAGCACAGGGATTCCCTCAGT. Two cycles of CT value difference (4-fold) was used as cutoff. The expression of DERPC was compared between the paired tumor and normal samples from the same individual.

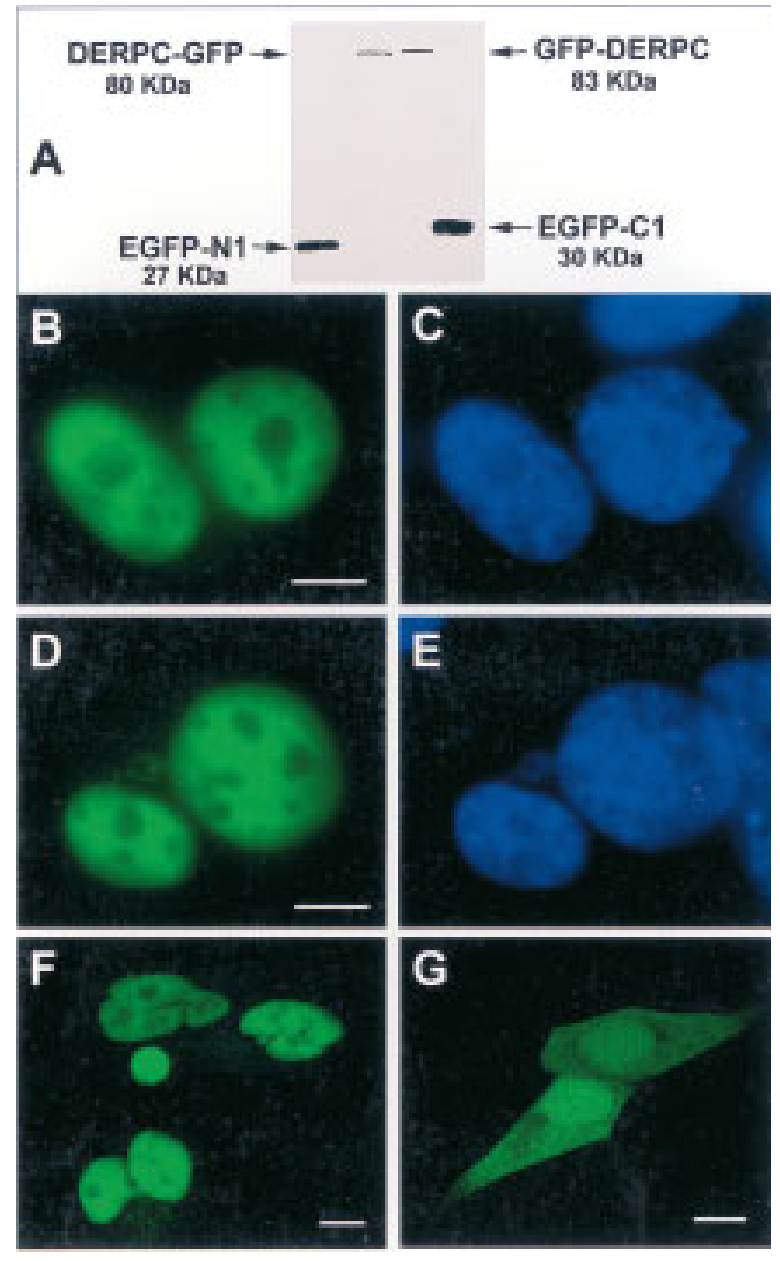

Fig. 4. Western blotting and cellular localization of DERPC in transiently transfected cells. (A) PC3 cells were transfected with both GFP-tagged DERPC constructs for $48 \mathrm{hr}$ and collected for blot analysis. DERPC-GFP and GFP-DERPC were detected with anti-GFP antibody for the predicted molecular mass ( 80 and $83 \mathrm{kDa}$, respectively) of the GFP (27 kDa for pEGFP-N1 or $30 \mathrm{kDa}$ for pEGFP-Cl) fused with DERPC (predicted size $51 \mathrm{kDa})$. PC3 cells (B, C, D, E) and LNCaP cells $(\mathbf{F}, \mathbf{G})$ were transfected with GFP-DERPC (B, C, F), DERPCGFP (D, E), and pEGFP-Nl (G) for $72 \mathrm{hr}$ and fixed by $3 \%$ paraformaldehyde then stained with DAPI (C, E). Both DERPC chimeras (B, D, F) were present diffusely in nucleus counterstained with DAPI (B, D) in cells transfected with either GFPDERPC (B, F) or DERPC-GFP constructs (D). DERPC was not found in nucleoli $(\mathbf{B}, \mathbf{D}, \mathbf{F})(\mathrm{bar}=10 \mu \mathrm{m}[\mathbf{B}-\mathbf{G}])$.

multi-tumor tissue array. Despite small sample size, reduced tumor-specific expression was remarkable in renal tumor tissues compared with the normal counterpart. This finding provided a strong indication of the possible involvement of DERPC in renal tumors. Because of the limited size of samples, further study is necessary to establish the correlation of DERPC expression and renal tumors.

In prostate tumors, LCM-derived RNA samples were used to assess the expression changes of DERPC by quantitative RT-PCR. We found that the prostate tumors exhibited decreased expression of DERPC in 

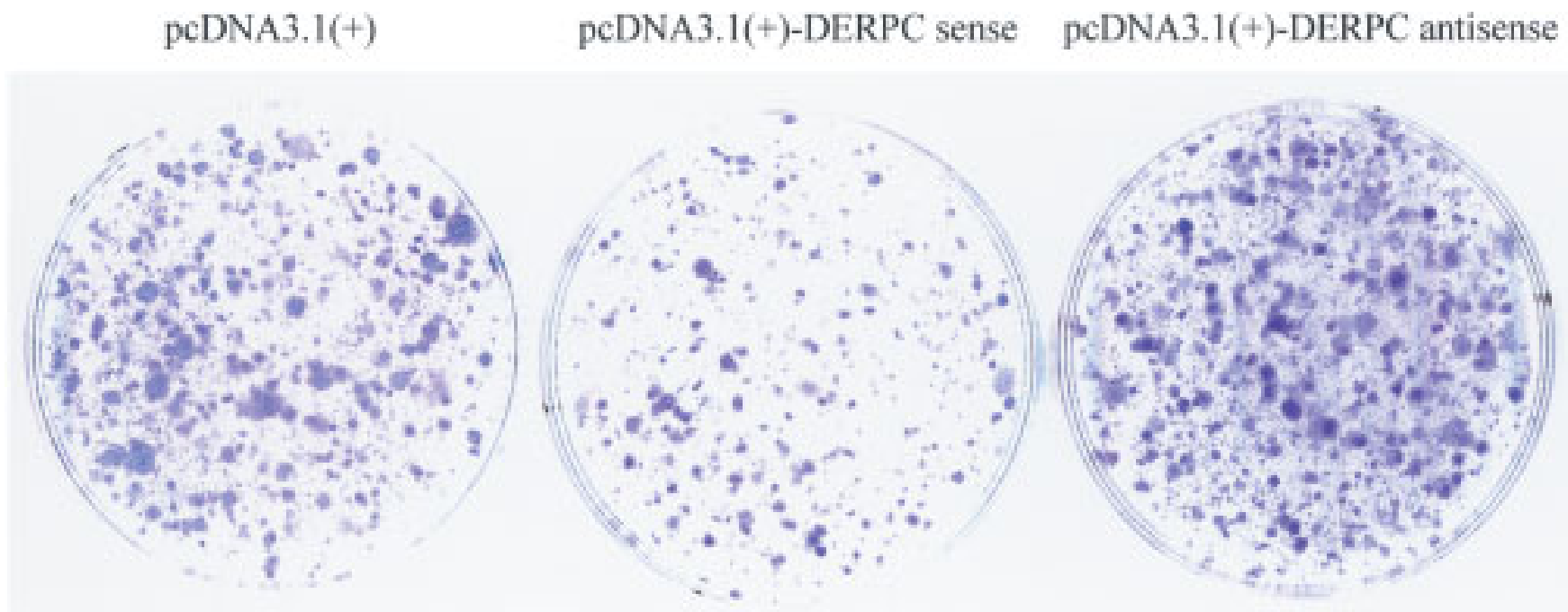

Fig. 5. Overexpression of DERPC inhibits colony formation of prostate cancer PC3 cells. PC 3 cells $\left(2 \times 10^{5}\right.$ cells) were plated in a $60 \mathrm{~m}$ culture dish for $24 \mathrm{hr}$ and incubated at $37{ }^{\circ} \mathrm{C}$. For transfection, $5 \mu \mathrm{g}$ each of pcDNA3.1(+), pcDNA-DERPC (sense), or pcDNA-DERPC(antisense) was transfected using TransFast reagent (Promega) according to the protocols provided. After 24 hr, cells were cultured in medium containing $400-500 \mu \mathrm{g} / \mathrm{ml}$ of geneticin (G-418) for 2-4 weeks. Cell colonies were stained with crystal violet (1 $\mathrm{mg} / \mathrm{ml}$ ).

approximately one third of the cases. The E-cadherin gene encoded an epithelial cell adhesion molecule whose decreased expression was frequently found in prostate tumors and was associated with poor prognosis in patients with CaP (32). The colocalization of DERPC with E-Cadherin on the frequent $\mathrm{LOH}$ region implies that expression of DERPC may undergo the same disruption as E-cadherin in cancer cells. The finding that only $32 \%$ of the 38 cases of prostate tumors showed down-regulated expression was not surprising, given the nature of highly expressed heterogeneity of prostate tumors. With the exception of the recently identified prostate tumor markers AMACR and DD3, which are evenly overexpressed in prostate tumors (33-35), a heterogeneous expression pattern of tumor-associated genes are commonly found in prostate tumors. The DNA sequence of DERPC was also analyzed in three common prostate tumor cell lines and 10 DNA samples of prostate tumors. No mutations were found (data not shown). It is likely that mutation of DERPC is rare in prostate tumors. It is the decreased expression of DERPC that possibly contributes to the tumor development in portions of CaP. Because the sample size in this initial study is relatively small, more detailed analysis with larger set of samples is needed to evaluate whether decreased expression of DERPC may be a biomarker for renal and prostate tumor. Development of antibody against DERPC will facilitate the study of DERPC expression in human cancers.

We have demonstrated that DERPC has a diffuse nucleoplasmic localization by fusing GFP at the $\mathrm{N}$ - or C-termini of DERPC. Most transcription factors are classified as proteins with a diffuse nucleoplasmic distribution (36). DERPC contains a proline-rich domain with abundant proline residues $(20 \%)$, a feature found in many proteins that are involved in protein-protein interactions. DERPC also contains 11 repeats of the sequence PXXP. This motif has been shown to play a role in signal transduction by interacting with $\mathrm{SH} 3$ domain containing proteins $(37,38)$. All high-affinity SH3 binding proteins contain this motif, and their binding specificity is conferred by the variable residues found within and flanking this consensus PXXP sequence. The presence of this motif in DERPC suggests that the protein might function as a binding protein of $\mathrm{SH} 3$ domain-containing proteins and could be involved in regulation or modification of these binding partners, many of which play important roles in the growth factor and signal transduction pathways $(39,40)$. It is tempting to think that DERPC may interfere with growth factor or stress-mediated signal transduction pathways by interacting with $\mathrm{SH} 3$ domain containing molecules. Preliminary data showing cell growth inhibitory effects of DERPC suggests its potential role in negative regulation of cell growth and loss of such function could favor tumorigenesis. Further study will address the potential biochemical function of DERPC in signal transudation and cell growth.

\section{Acknowledgments}

We thank Ms Justine Cowan for her critical reading of the manuscript. This work was supported by the Center for Prostate Disease Research, a program of the Henry M. Jackson Foundation for the Advancement of Military Medicine (Rockville, MD), funded 
by the United States Army Medical Research and Material Command.

The author is supported by a grant from the Center for Prostate Disease Research, a program of the Henry M. Jackson Foundation for the Advancement of Military Medicine (Rockville, MD), funded by the U.S. Army Medical Research and Materiel Command.

\section{References}

1. Jemal A, Thomas A, Murray T, Thun M. (2002) Cancer statistics, 2002. Cancer J. Clin. 52: 23-47.

2. Cabeza-Arvelaiz Y, Sepulveda JL, Lebovitz RM, Thompson TC, Chinault AC. (2001) Functional identification of LZTS I as a candidate prostate tumor suppressor gene on human chromosome 8p22. Oncogene 20: 4169-4179.

3. Baffa R, Santoro R, Bullrich F, Mandes B, Ishii H, Croce CM. (2000) Definition and refinement of chromosome $8 p$ regions of loss of heterozygosity in gastric cancer. Clin. Cancer Res. 6: 1372-1377.

4. Sato K, Qian J, Slezak JM, et al. (1999) Clinical significance of alterations of chromosome 8 in high-grade, advanced, nonmetastatic prostate carcinoma. J. Natl. Cancer Inst. 91: 1574-1580.

5. Dahiya R, Perinchery G, Deng G, Lee C. (1998) Multiple sites of loss of heterozygosity on chromosome 8 in human breast cancer has differential correlation with clinical parameters. Int. J. Oncol. 12: 811-816.

6. Voeller HJ, Augustus M, Madike V, Bova GS, Carter KC, Gelmann EP. (1997) Coding region of NKX3.1, a prostate-specific homeobox gene on $8 \mathrm{p} 21$, is not mutated in human prostate cancers. Cancer Res. 57: 4455-4459.

7. Yaremko ML, Kutza C, Lyzak J, Mick R, Recant WM, Westbrook CA. (1996) Loss of heterozygosity from the short arm of chromosome 8 is associated with invasive behavior in breast cancer. Genes Chromosomes Cancer 16: 189-195.

8. Li J, Yen C, Liaw D, et al. (1997) PTEN, a putative protein tyrosine phosphatase gene mutated in human brain, breast, and prostate cancer. Science 275: 1943-1947.

9. Suzuki H, Freije D, Nusskern DR, et al. (1998) Interfocal heterogeneity of PTEN/MMACl gene alterations in multiple metastatic prostate cancer tissues. Cancer Res. 58: 204209.

10. Zenklusen JC, Conti CJ, Green ED. (2001) Mutational and functional analyses reveal that ST7 is a highly conserved tumor-suppressor gene on human chromosome 7q31. Nat. Genet. 27: 392-398.

11. Narla G, Heath KE, Reeves HL, et al. (2001) KLF6, a candidate tumor suppressor gene mutated in prostate cancer. Science 294: 2563-2566.

12. Reiter RE, Sato I, Thomas G, et al. (2000) Coamplification of prostate stem cell antigen (PSCA) and MYC in locally advanced prostate cancer. Genes Chromosomes Cancer 27: 95103.

13. Cher ML, Bova GS, Moore DH, et al. (1996) Genetic alterations in untreated metastases and androgen-independent prostate cancer detected by comparative genomic hybridization and allelotyping. Cancer Res. 56: 3091-3102.

14. Sun M, Ma L, Li J, et al. (2002) Characterization of a novel tumor suppressor gene locus on chromosome 6q16.1 in prostate cancer. Proceedings of the American Association for Cancer Research 43: 630 .

15. Cussenot O, Valeri A, Berthon P, Fournier G, Mangin P. (1998) Hereditary prostate cancer and other genetic predispositions to prostate cancer. Urol. Int. 60(suppl 2): 30-34.

16. Isaacs WB. (1995) Molecular genetics of prostate cancer. Cancer Surv. 25: 357-379.

17. Tavtigian SV, Simard J, Teng DH, et al. (2001) A candidate prostate cancer susceptibility gene at chromosome 17p. Nat. Genet. 27: 172-180.
18. Carpten J, Nupponen N, Isaacs S, et al. (2002) Germline mutations in the ribonuclease $\mathrm{L}$ gene in families showing linkage with HPC1. Nat. Genet. 30: 181-184.

19. Rokman A, Ikonen T, Seppala EH, et al. (2002) Germline alterations of the RNASEL gene, a candidate HPCl gene at 1q25, in patients and families with prostate cancer. Am. J. Hum. Genet. 70: 1299-1304.

20. Latil A, Cussenot O, Fournier G, Driouch K, Lidereau R. (1997) Loss of heterozygosity at chromosome $16 \mathrm{q}$ in prostate adenocarcinoma: identification of three independent regions. Cancer Res. 57: 1058-1062.

21. Li C, Berx G, Larsson C, et al. (1999) Distinct deleted regions on chromosome segment 16q23-24 associated with metastases in prostate cancer. Genes Chromosomes Cancer 24: 175-182.

22. Hainsworth PJ, Raphael KL, Stillwell RG, Bennett RC, Garson OM. (1991) Cytogenetic features of twenty-six primary breast cancers. Cancer Genet. Cytogenet. 53: 205-218.

23. Callen DF, Crawford J, Derwas C, Cleton-Jansen AM, Cornelisse CJ, Baker E. (2002) Defining regions of loss of heterozygosity of $16 \mathrm{q}$ in breast cancer cell lines. Cancer Genet. Cytogenet. 133: 76-82.

24. Dome JS, Coppes MJ. (2002) Recent advances in Wilms tumor genetics. Curr. Opin. Pediatr. 14: 5-11.

25. Guan XY, Fang Y, Sham JS, et al. (2000) Recurrent chromosome alterations in hepatocellular carcinoma detected by comparative genomic hybridization. Genes Chromosomes Cancer. 29: 110-116.

26. Horwitz M, Benson KF, Li FQ, et al. (1997) Genetic heterogeneity in familial acute myelogenous leukemia: evidence for a second locus at chromosome 16q21-23.2. Am. J. Hum. Genet. 61: 873-881.

27. Paris PL, Witte JS, Kupelian PA, et al. (2000) Identification and fine mapping of a region showing a high frequency of allelic imbalance on chromosome 16q23.2 that corresponds to a prostate cancer susceptibility locus. Cancer Res. 60: 3645-3649.

28. Ikonen T, Matikainen M, Mononen N, et al. (2001) Association of E-cadherin germ-line alterations with prostate cancer. Clin. Cancer Res. 7: 3465-3471.

29. Graff JR, Herman JG, Lapidus RG, et al. (1995) E-cadherin expression is silenced by DNA hypermethylation in human breast and prostate carcinomas. Cancer Res. 55: 5195-5199.

30. Corn PG, Smith BD, Ruckdeschel ES, Douglas D, Baylin SB, Herman JG. (2000) E-cadherin expression is silenced by $5^{\prime}$ CpG island methylation in acute leukemia. Clin. Cancer Res. 6: 4243-4248.

31. Xu LL, Su YP, Labiche R, et al. (2001) Quantitative expression profile of androgen-regulated genes in prostate cancer cells and identification of prostate-specific genes. Int. J. Cancer 92: 322-328.

32. Umbas R, Isaacs WB, Bringuier PP, et al. (1994) Decreased Ecadherin expression is associated with poor prognosis in patients with prostate cancer. Cancer Res. 54: 3929-3933.

33. Luo J, Zha S, Gage WR, et al. (2002) Alpha-methylacyl-CoA racemase: a new molecular marker for prostate cancer. Cancer Res. 62: 2220-2226.

34. Rubin MA, Zhou M, Dhanasekaran SM, et al. (2002) alphaMethylacyl coenzyme A racemase as a tissue biomarker for prostate cancer. JAMA 287: 1662-1670.

35. de Kok JB, Verhaegh GW, Roelofs RW, et al. (2002) DD3(PCA3), a very sensitive and specific marker to detect prostate tumors. Cancer Res. 62: 2695-2698.

36. Bickmore WA, Sutherland HGE. (2002) Addressing protein localization within the nucleus. EMBO J. 21: 1248-1254.

37. Feller SM, Ren R, Hanufusa H, Baltimore D. (1994) SH2 and $\mathrm{SH} 3$ domains as molecular adhesives: the interactions of Crk and Abl. Trends Biochem. Sci. 19: 453-458.

38. Cohen GB, Ren R, Baltimore D. (1995) Modular binding domains in signal transduction proteins. Cell 80: 237-248.

39. Schlessinger J. (1994) $\mathrm{SH} 2 / \mathrm{SH} 3$ signaling proteins. Curr. Opin. Genet. Dev. 4: 25-30.

40. Birge RB, Knudsen BS, Besser D, Hanafusa H. (1996) SH2 and $\mathrm{SH} 3$-containing adaptor proteins: redundant or independent mediators of intracellular signal transduction. Genes Cells 1: 595-613. 